\title{
Relato PET-Saúde/GraduaSUS: trajetória da Odontologia em uma instituição privada, 2016-2017
}

\author{
Patrícia Suguri Cristino*; Mônica Moura Costa e Silva**; Tatiana Frederico de Almeida***; Haline \\ Souza Antunes****; Ticiane Teixeira de Mendonça******
}

* Mestre em Odontologia Restauradora, Escola Bahiana de Medicina e Saúde Pública e Universidade Federal da Bahia

** Mestre em Saúde Comunitária, Escola Bahiana de Medicina e Saúde Pública e Secretaria Estadual de Saúde da Bahia

*** Doutora em Saúde Pública, Universidade Federal da Bahia e Secretaria Municipal de Saúde de Salvador/BA

**** Mestre em Saúde Coletiva, Secretaria Municipal de Saúde de Salvador/BA

***** Especialista em Saúde Coletiva, Secretaria Municipal de Saúde de Salvador/BA

Recebido em 11/02/2018. Aprovado em 09/12/2018.

\begin{abstract}
RESUMO
Este estudo refere-se a um relato de experiência do primeiro ano de vigência do PETSaúde/GraduaSUS do curso de Odontologia da Escola Bahiana de Medicina e Saúde Pública, uma instituição do ensino superior privada, em Salvador/BA. Quanto às ações desenvolvidas, destacamse as atividades relacionadas com as mudanças curriculares e aquelas voltadas à integração ensinoserviço. Sobre os resultados já alcançados, verificam-se mudanças na formação dos discentes, inseridos na realidade da atenção primária no SUS; formação de preceptores em saúde bucal com habilidades e competências que favorecem a formação de profissionais mais críticos, reflexivos, humanizados e preparados para oferecer Atenção Primária à Saúde.

Descritores:Atenção Primária à Saúde. Integração Docente-Assistencial. Odontologia.
\end{abstract}

\section{INTRODUÇÃO}

No Brasil, observa-se descompasso da formação do profissional de saúde, incluindo o cirurgião-dentista, em relação às políticas e ações que direcionam os serviços públicos de saúde, que atingem a maioria da população deste país, ocasionando consequências desastrosas para o sistema de serviços de saúde e para o perfil 
sanitário da população. Constata-se o desequilíbrio entre oferta de serviços e demanda de profissionais, a má-distribuição destes e dificuldades de fixação, o desconhecimento e falta de habilidades e competências para assistir às reais necessidades de saúde das pessoas e a proposição de estratégias equivocadas de gestão da saúde ${ }^{1}$. Desse modo, são reais as lacunas na relação entre os serviços de saúde e o ensino/formação em saúde.

Desde a criação do Sistema Único de Saúde (SUS), em 1988, muitos avanços ocorreram em direção à integralidade, universalidade e equidade, princípios doutrinários deste sistema. Porém estes avanços poderiam ter sido mais significativos, pois ainda são expressivas as resistências de profissionais a mudanças nos processos de trabalho, a tendência ao trabalho fragmentado e a valorização das super especialidades na realidade da Atenção Primária à Saúde (APS) ${ }^{2}$.

As Diretrizes Curriculares Nacionais (DCN) foram elaboradas em 2002 para o ensino de graduação em Odontologia e definem os princípios, fundamentos, condições e procedimentos da formação de cirurgiõesdentistas, estabelecidas pela Câmara de Educação Superior do Conselho Nacional de Educação (CNE), para aplicação em âmbito nacional na organização, desenvolvimento e avaliação dos projetos pedagógicos dos cursos de graduação em Odontologia. As DCN preconizam a formação na graduação de um profissional de saúde crítico, reflexivo, preparado para atuar em equipe e na realidade do mercado de trabalho, exigindo experiências de ensino-aprendizagem diferenciadas no contexto do SUS. Segundo Alves $(2005)^{2}$, os cenários de trabalho no SUS e no mercado em geral sofrem processos de mudanças contínuas, e a APS passou a ser um cenário primordial na formação desse novo profissional.

Os Ministérios da Saúde (MS) e da Educação (MEC), compreendendo a necessidade de atuarem como facilitadores da integração entre ensino e serviços de saúde para a adequada implantação do SUS, têm promovido iniciativas com o intuito de estimular a aproximação das Universidades dos serviços de saúde da APS, como o Pro-Med, o ProSaúde, o PET-Saúde, entre outros.

Em setembro de 2015, o MS, por intermédio da Secretaria de Gestão do Trabalho e da Educação na Saúde (SGTES), lançou o edital do PETSaúde/GraduaSUS, selecionando projetos que envolviam a participação de Secretarias Estaduais ou Municipais de Saúde, assim como Instituições do Ensino Superior (IES) que desenvolvessem: mudanças curriculares alinhadas às DCN para todos os cursos de graduação na área da saúde; qualificação dos processos de integração ensinoserviço-comunidade de forma articulada entre o SUS e as instituições de ensino; articulação com projetos do MS e MEC e/ou outros projetos de âmbito local ou regional relacionados à integração ensino-serviço-comunidade ${ }^{3}$.

Uma das IES selecionadas para o PETSaúde/GraduaSUS foi a Escola Bahiana de Medicina e Saúde Pública (EBMSP), localizada em Salvador/BA, uma IES privada, com 65 anos de dedicação à formação de profissionais na área de saúde. Participam do PET-Saúde/GraduaSUS, vigente no período de 2016 e 2017 na EBMSP, os cursos de Odontologia, Enfermagem e Medicina e a Secretaria Municipal de Saúde (SMS) de Salvador/BA, que atua como coordenadora geral e permite a participação de preceptores do serviço para contribuírem com a execução e planejamento do projeto. Estudantes de graduação de cada um destes cursos participam do PET como bolsistas ou voluntários, além de três docentes de cada curso, que atuam como tutoras, sendo uma delas coordenadora do PET. É válido informar que o curso de Odontologia da EBMSP possui 19 anos e foi o primeiro curso de uma IES privada no estado.

O projeto do PET-Saúde/GraduaSUS da EBMSP para o período de 2016 e 2017 possui dois eixos de intervenção: Mudanças Curriculares (Eixo 
1) e Integração Ensino-Serviço (Eixo 2).

Este artigo foi escrito como um relato de experiência considerando as práticas da EBMSP, com destaque para a trajetória do curso de Odontologia durante o primeiro ano de vigência do projeto, de maio de 2016 a junho de 2017. O seu objetivo é descrever como se desenvolveu o projeto neste curso, a partir do olhar de professoras tutoras, alunos e preceptores que participaram da implantação e da execução do projeto, refletindo criticamente a respeito das intervenções no serviçocomunidade e no ensino.

Para isto foi realizada uma revisão da literatura nos bancos de dados SciELO, LILACS, BIREME, BVS, MEDLINE, no período de 2002 a 2017, em busca de estudos relacionados com a temática de integração ensino-serviço de saúde e mudanças curriculares; a equipe envolvida com o projeto reuniu-se algumas vezes e discutiu as experiências vivenciadas, cujos registros estão aqui relatados e analisados criticamente à luz de aspectos teóricos da literatura pesquisada. Ressaltase que não há restrições quanto aos aspectos éticos, uma vez que não foi utilizado nenhum tipo de dado ou de experiência interpessoal com seres humanos.

\section{RELATO DE EXPERIÊNCIA}

\subsection{Mudanças Curriculares (Eixo 1)}

Assim que o PET-Saúde/GraduaSUS foi implementado no curso de Odontologia da EBMSP em maio de 2016, os estudantes (bolsistas e voluntários), sob a orientação das tutoras, elaboraram seminários para a discussão de temas relacionados com a construção do Currículo Acadêmico. Foram constituídos três grupos que discutiram as temáticas "DCN", "Princípios de organização do currículo" e "Experiências curriculares exitosas em Odontologia".

Este momento teve como intuito permitir uma maior compreensão de todo o grupo (professora coordenadora, professoras tutoras, preceptores do serviço e estudantes) a respeito da importância da formação curricular com base nas DCN, em busca de uma formação em Odontologia condizente com a realidade social e sanitária da população brasileira. Ele também contribuiu para instrumentalizar o grupo quanto aos princípios da construção curricular e para apresentar estratégias de ensino, pesquisa e extensão bem sucedidas em Odontologia, as quais tinham como foco uma formação mais integral, humanizada, crítica e reflexiva, conforme preconizado pelas $\mathrm{DCN}^{4}$.

O sistema educacional brasileiro é constituído por uma variedade de desenhos curriculares e práticas pedagógicas desarticulados da realidade social e desconectados de outras disciplinas. Na formação em saúde predominam currículos de conteúdos sequenciais, que pouco valorizam as possibilidades de construção de trajetórias educacionais e experienciais singulares. O PET procura proporcionar aos alunos vivências que adotam a interdisciplinaridade, proporcionando a entrada dos alunos cada vez mais cedo aos cenários de prática ${ }^{5}$. De acordo com Feuerwerker e Sena $(2002)^{6}$, as características fundamentais de uma proposta de mudança/inovação curricular são: organização curricular em módulos integrados, baseados em problemas extraídos da realidade; metodologias ativas de ensino-aprendizagem; prática nos serviços, interação com a comunidade desde o início do curso e avaliação permanente do processo.

A fim de possibilitar um diagnóstico inicial quanto a adequação às DCN no que tange os princípios e diretrizes do SUS e à APS na formação curricular do curso de Odontologia, foi realizada uma análise de todos os planos de ensino dos componentes curriculares deste curso. Um grupo de 06 alunos orientado por uma das professoras tutoras do PET-Saúde/GraduaSUS realizou esta análise de forma sistemática com base em uma matriz estruturada, construída especialmente para 
este fim. A partir das DCN de Odontologia, tópicos diretamente relacionados com o SUS e com a APS foram selecionados e cada plano de ensino foi analisado. Foram registradas quatro possíveis situações para cada tópico: "Contemplado"; "Não contemplado", "Contemplado parcialmente", "Não se aplica a este componente".

Ao final desta análise foram observadas algumas potencialidades do curso de Odontologia da EBMSP, descritas nos planos de ensino, tais como: a ênfase no trabalho em equipe; a formação de profissionais com perfil proativo e empreendedor; a ênfase na formação de profissionais com liderança; a utilização de metodologias ativas de aprendizagem; a realização de procedimentos odontológicos adequados para prevenção, tratamento e controle das principais doenças e agravos bucais; o estímulo à investigação científica; a promoção de atividades que desenvolvem habilidades intelectuais; a introdução do aluno precocemente com o conteúdo prático. Alguns autores enfatizam que as atividades que buscam a integração teoria- prática, o trabalho em equipe e a integralidade da atenção em saúde potencializam o aprendizado, a reorganização do modelo de atenção e o enfrentamento das reais necessidades sanitárias da população ${ }^{7,8}$.

Porém, algumas fragilidades foram apontadas. Os planos de ensino dos componentes fazem pouca referência ao SUS como cenário prioritário de atuação profissional e à atenção integral à saúde, em um sistema de referência e contra-referência; constatou-se pouca referência à determinação social do processo saúde-doença, considerando a integralidade da atenção à saúde $\mathrm{e}$ o paradigma da Promoção da Saúde enquanto modelo de atenção; alguns componentes ainda enfatizam pouco a importância da formação generalista, humanística e ética, promotora da saúde integral do paciente, atenta às necessidades individuais e coletivas; a falta de valorização da atuação em equipes interprofissionais, interdisciplinares e transdisciplinares. Ceccim e Ferla $(2008)^{9}$ alertam que não se concebem mais processos educativos cujo único cenário de práticas seja o hospital universitário, pois é necessário romper com a dissociação entre clínica e política, o que é possível apenas em ambientes e situações reais. A educação deve priorizar o SUS enquanto cenário de práticas, que possibilita a realização de vivências essenciais ao aprendizado.

Esta análise diagnóstica dos planos de ensino do curso de Odontologia deverá embasar as revisões dos planos de ensino, que serão executadas pelo grupo de docentes de cada componente no ano de 2018. Ademais, também é importante frisar que a revisão de planos de ensino para questões relativas à APS como porta de entrada do SUS, à integralidade da atenção à saúde e à formação generalista, ética e reflexiva são apenas um mecanismo para uma atuação na prática docente formativa que valorize e agregue estes valores e princípios no cotidiano do ensino.

Conforme proposto pela Política Nacional de Educação Permanente em Saúde (PNEPS), lançada em 2003, a rede pública de saúde deve constituir-se em um espaço de ensinoaprendizagem no exercício do trabalho, o que significa que a formação dos estudantes e profissionais deve acontecer dentro do próprio serviço, em situações reais no $\mathrm{SUS}^{10}$. O ensino no cenário da APS oferece ao estudante, em busca de melhor qualificação profissional, uma melhor assistência ao usuário, sua família e à comunidade ${ }^{11}$.

No estudo de Haddad et al.(2006) ${ }^{12}$, verificou-se que a interdisciplinaridade representa uma das principais dificuldades apontadas na implantação de currículos inovadores em saúde. É preciso que se conheçam quais as concepções dos docentes e gestores acadêmicos sobre o currículo, integração ensino-serviço. Os autores ressaltaram que esse é um dos pontos que dificultam a aderência às DCN. O processo de mudança 
curricular deve ser realizado de forma organizada, sendo necessária uma equipe condutora e o compromisso docente para com avanços em uma prática educativa de qualidade, criadora de possibilidades de intervenção crítica ${ }^{13}$.

Os componentes curriculares denominados "Saúde Coletiva IV" e "Saúde Coletiva V" direcionados a alunos do $9^{\circ}$ e $10^{\circ}$ semestres correspondem ao Estágio Supervisionado em campos de prática do SUS. Tradicionalmente estes componentes costumavam direcionar os alunos para vivências de saúde bucal em instituições privadas e públicas, dentre estas havia a possibilidade de inserção dos alunos em serviços de pronto atendimento e de urgência odontológica, que não possibilitavam uma experiência real da APS, tendo a Estratégia Saúde da Família como porta de entrada do sistema.

Assim, após alguns encontros entre representantes da SMS de Salvador/BA, da EBMSP, inclusive da professora Coordenadora do PET-Saúde/GraduaSUS, seus preceptores e tutoras, um novo modelo de formação para o Estágio Supervisionado foi planejado e encontra-se em fase de implantação na EBMSP. De acordo com este novo modelo, todos os alunos do $10^{\circ}$ semestre desenvolverão suas atividades de Estágio em Unidades de Saúde da Família (USF) ou Unidades Básicas de Saúde tradicionais (UBS) dos distritos sanitários do Cabula-Beiru, Brotas e Pau da Lima do município de Salvador durante dois turnos semanais e serão orientados por preceptores cirurgiões-dentistas do local. Desse modo, eles terão a oportunidade de ainda na graduação vivenciarem o funcionamento da APS estruturada de maneira mais integral e humanizada no SUS real. Estes profissionais chegarão ao mercado de trabalho mais preparados na teoria e na prática para enfrentarem os desafios da atuação no setor público. Inicialmente pensou-se apenas nas USF como cenário de prática, mas não contemplaria o total de estudantes.
Os Estágios Supervisionados em Saúde Coletiva proporcionam a discussão da problemática de saúde da população para o discente, a reflexão sobre o cotidiano das pessoas (como vivem e determinantes da saúde e da doença). $\mathrm{O}$ aprendizado, com base na realidade da comunidade, traz significação às ações de saúde e um aprendizado vivo e transformador da formação em saúde ${ }^{14}$.

Vale salientar que a receptividade e adesão por parte dos cirurgiões-dentistas da SMS foram positivas. Preocupada com a formação para a atuação como preceptores destes dentistas, a equipe coordenadora do PET-Saúde/GraduaSUS do curso de Odontologia da EBMSP planejou um "Curso de Extensão para formação de preceptores no SUS", desenvolvido de fevereiro a junho de 2017, com duração de cinco meses. O público-alvo foi formado por cirurgiões-dentistas que atuam como preceptores dos estudantes do $10^{\circ}$ semestre do curso de Odontologia da EBMSP em USF e UBS de Salvador.

As vivências possibilitadas nos serviços de saúde com base na atuação da preceptoria representam em sua essência uma prática muito almejada pelos pesquisadores da Educação em Saúde, que é a integração entre os serviços de saúde e a academia ${ }^{15}$. Esta integração é estabelecida a partir de interesses e necessidades identificados e percebidos de ambos os lados. Por um lado, os alunos inseridos no SUS beneficiam-se com o conhecimento compartilhado pelos preceptores, os quais têm a expectativa de aproveitar as oportunidades de ampliação da sua formação, a partir da inserção em cursos de formação complementar oferecidos pela academia ${ }^{14}$.

Este curso de formação de preceptores oferecido pela EBMSP teve um momento presencial, constituído por Oficinas e Rodas de Conversa no campus da instituição. Este momento ocorreu durante um dia por mês, sendo que as Oficinas foram planejadas para o turno da manhã, 
quando diversas temáticas foram trabalhadas pelo grupo de dentistas, coordenados por professoras que atuam no PET e por professores do Estágio Supervisionado. As temáticas abordadas foram: "O papel da Preceptoria", "Acolhimento", "Planejamento e Programação Local em Saúde", "Enfoque epidemiológico no SUS", "Tecnologias preventivas em saúde bucal" e "Bioética". No turno da tarde aconteceram "Rodas de Conversa"envolvendo alunos do Estágio Supervisionado e seus respectivos preceptores, professores do componente curricular e professoras que atuam no PET-Saúde/GraduaSUS. Este momento foi oportuno para troca de experiências, levantamento de avanços e dificuldades nos campos de prática, busca de soluções compartilhadas e revisão de conteúdos da Saúde Coletiva.

Uma complementação deste curso ocorreu através da tecnologia da "Educação à Distância" (EAD), possibilitada pelo uso da plataforma moodle da EBMSP. Assim, no momento EaD, os dentistas complementaram teoricamente conteúdos trabalhados nas Oficinas por meio da leitura de textos e artigos, visualização de aulas on line, documentários, filmes e da realização de atividades relacionadas com a temática em questão. A professora coordenadora do PETSaúde/GraduaSUS e as duas professoras tutoras atuaram neste momento como tutoras das atividades EaD, orientando mais de perto cerca de 10 dentistas cada uma.

A EBMSP e o PET-Saúde/GraduaSUS reconhecem a importância desta formação dos dentistas para atuarem como preceptores da graduação em Odontologia no serviço público, uma vez que o ensino exige o desenvolvimento de competências e habilidades que nem todos os profissionais desenvolvem ao longo de sua carreira. Além disso, as "Rodas de Conversa" permitem a criação de novos caminhos para o estabelecimento de uma comunicação verdadeiramente dialógica e democrática entre uma instituição de ensino e de serviço de saúde.

\subsection{Integração Ensino-Serviço (Eixo 2)}

$\mathrm{O}$ início das atividades do PETSaúde/GraduaSUS na EBMSP foi marcado pela participação no "Acolhimento Pedagógico": todos os alunos do PET da EBMSP (Medicina, Enfermagem e Odontologia) foram convidados para participar de uma visita técnica à SMS, com o intuito que fossem apresentadas a organização e estrutura da instituição. Os alunos envolvidos neste processo estavam melhor preparados para a inserção nas atividades propostas pelo PET em busca de maior integração ensino-serviço.

Originalmente, o Acolhimento Pedagógico da SMS de Salvador consiste numa atividade de Educação Permanente junto aos profissionais de saúde recém contratados pela instituição. Foi proposto pela Coordenação Geral do PETSaúde/GraduaSUS que os atores desse projeto organizassem e conduzissem alguns ciclos de oficinas pedagógicas em 4 unidades de saúde (1 UBS e 3 USF) com os propósitos de Educação Permanente e de estreitamento entre a IES e esses campos de prática (todas são unidades que já recebem alunos da EBMSP).

Os estudantes e professores (coordenadora e tutoras) participantes do PET também foram convidados em diversas oportunidades para colaborarem em ações de saúde programadas pelas USF. Os alunos também contribuíram para a construção de um levantamento dos recursos humanos e dos seus processos de trabalho em USF dos distritos sanitários do Cabula-Beiru e de Brotas.. Durante este levantamento, os discentes permaneceram por um período no interior das USF vivenciando o dia a dia da APS no SUS.

No processo de integração ensino-serviço, uma das vantagens é que o estudante traz inovação ao trabalho em saúde, além de estimular, no preceptor/profissional, a necessidade de educação 
permanente para a qualificação do cuidado em saúde ${ }^{14}$.

Outra forma de integração do ensino e serviço que o PET-Saúde/GraduaSUS vem possibilitando é através da realização de atividades práticas de saúde bucal coletiva de alguns componentes curriculares da EBMSP em áreas de abrangência de USF. O componente de Saúde Coletiva III vem desenvolvendo inquéritos epidemiológicos de saúde bucal e atividades preventivas junto a escolares do Cabula-Beiru; os componentes curriculares Saúde Coletiva I e II realizam atividades de territorialização e acolhimento neste mesmo distrito sanitário. As intervenções no campo do SUS são transformadoras no processo da educação universitária, permitindo a aproximação do discente com o contexto social, político e econômico da população brasileira.

O grupo do PET vem participando dos eventos científicos promovidos pela EBMSP, a exemplo da Mostra Científica e Cultural em outubro de 2016, quanto foi organizada uma intervenção em saúde do trabalhador desta instituição. Os colaboradores presentes na Mostra puderam participar de atividades educativas e preventivas de saúde geral e bucal conduzidas por estudantes bolsistas e voluntários treinados e supervisionados por professores do PET. Ademais, resultados do PET Gradua SUS e experiências exitosas de integração ensino-serviço estão sendo divulgados em outros eventos científicos promovidos pela EBMSP, a exemplo da Jornada Odontológica da Bahiana (JOBA).

Vale destacar que o processo de Educação Permanente do corpo docente desta instituição de ensino também vem sendo possibilitado com a atuação do PET. Os professores de Odontologia, preceptores e tutores do PET participaram de rodas de conversa, com o intuito de que fossem apresentadas a visão dos preceptores sobre os estudantes da EBMSP que realizam Estágio
Supervisionado nas USF onde trabalham. Nesse momento, os professores deste curso também puderam se aproximar da rotina de trabalho das equipes de saúde bucal das USF e da gestão de saúde bucal deste município, uma vez que a coordenadora de saúde bucal de Salvador é preceptora do programa e deu seu depoimento sobre a situação atual de conformação da rede em termos de recursos humanos e estrutura física de unidades de saúde e do quadro epidemiológico de saúde bucal do município de Salvador, apresentando resultados do último inquérito de saúde bucal realizado em 2015.

Neta e Alves (2016) ${ }^{15}$ comentam que é necessário refletir sobre como transformar as situações pedagógicas em 'oportunidades de inovação no ensino da saúde'. É fundamental o desenvolvimento da capacidade de inovação, dinamização e problematização pelos docentes, transformando situações reais em 'momentos de rico aprendizado', e que eles não sejam limitados pelo currículo disciplinar nem acabem por atuar de maneira a promover pouca inovação. As rodas de conversa realizadas acima com professores da EBMSP caminham no sentido de proporcionar aos docentes uma visão mais realista das condições de saúde bucal da população soteropolitana. O reconhecimento disto pode contribuir para um processo de formação inovador e dinâmico.

Paralelamente às ações de ensino, extensão e intervenções no serviço de saúde e na EBMSP descritas acima, estão sendo desenvolvidos projetos de pesquisa que visam traçar um perfil detalhado dos trabalhadores da SMS e do seu processo de trabalho, atuantes em áreas dos distritos sanitários do Cabula-Beiru e de Brotas, onde estão localizados os campi da EBMSP, assim como relatos de experiências e pesquisas etnográficas de intervenções específicas.

\section{CONSIDERAÇÕES FINAIS}

A formação em Odontologia é, 
historicamente, marcada pelo foco no desenvolvimento da técnica, no reconhecimento das patologias bucais e na ênfase do trabalho no setor privado (clínicas odontológicas). Desse modo, um dos grandes desafios do ensino odontológico é a formação contextualizada com a realidade nacional, no que tange a organização dos serviços de saúde, assim como o reconhecimento do quadro epidemiológico da população e a capacidade de assistir esta população de forma humanizada e resolutiva. As DCN enfatizam a necessidade e importância de uma formação mais qualificada, com o intuito de abordar os sujeitos, famíliase a comunidade dentro de seu contexto socioeconômico e cultural, o que também exige mudança na forma do exercício da docência.

Nos últimos anos observam-se no país mudanças significativas na política de saúde bucal, que contribuíram para a maior inserção do cirurgião-dentista no setor público, atuando nas USF e também na atenção especializada, nos Centros de Especialidades Odontológicas. Por tudo isso, observamos uma forte necessidade de mudanças na formação e no perfil do egresso formado. Melhorias na integração entre o ensino em saúde com os serviços são necessárias, e a trajetória do curso de Odontologia do PETSaúde/GraduaSUS implantado na EBMSP vem apontando para caminhos bem sucedidos nesse sentido, tanto no que diz respeito às mudançascurriculares como à integração ensinoserviço propriamente dita.

Nesse processo, ressaltamos a presença de aspectos facilitadores como: o apoio da gestão das instituições envolvidas (EBMSP e SMS), assim como dos discentes, tutoras e preceptores participantes do PET e dos cirurgiões-dentistas da SMS, os quais demonstram-se envolvidos com a proposta e intervenções planejadas. Salienta-se a importância de divulgação desta experiência para o fortalecimento das relações entre o ensino e os serviços de saúde, o aprimoramento da formação profissional em busca de um SUS consolidado e comprometido com mudanças nas condições de vida e saúde do povo brasileiro.

\section{ABSTRACT}

This study refers to an experience report of the first year of PET-Saúde/GraduaSUS of the Dentistry course at Escola Bahiana de Medicina e Saúde Pública, a private higher education institution in Salvador/BA. As for the actions developed, the activities related to curricular changes and those focused on the teaching-service integration stand out. On the already achieved results, there are changes in the formation of the students, inserted in the reality of the primary care in SUS; training of oral health preceptors with skills and competencies that favor the formation of more critical, reflexive, humanized and attentive Primary Health Care professionals.

Descriptors: Primary Health Care. TeachingAssistance Integration. Dentistry

\section{REFERÊNCIAS}

1. Brasil. Ministério da Saúde. Secretaria de Gestão do Trabalho e da Educação na Saúde. A Educação e o Trabalho na Saúde: A Política e suas Ações. Brasília: Ministério da Saúde, 2009.

2. Alves VS. Um modelo de educação em saúde para o Programa de Saúde da família: pela integralidade da atenção e reorientação do modelo assistencial. Interface. 2009; 9 (16): 3952.

3. Brasil. Ministério da Saúde. Edital no - 13, de 28 de setembro de 2015.Seleção para o Programa de Educação para o Trabalho em saúde - PET-SAÚDE/GRADUASUS 2016/2017, 2015.

4. Brasil. Resolução CNE/CES n ${ }^{\circ} 3$, de 19 de fevereiro de 2002. Diário Oficial da República Federativa do Brasil, Brasília, 4 mar 2002, seção 1, p. 10.

5. Santos CG,Portugal FTA, Silva MAB, Souza AC, Abrahão AC. Formação em saúde e produção de vínculo:uma experiência PET- 
Saúde na rede de Niterói, RJ, Brasil. Interface Comunic Saúde Educ. 2015; 19(1): 985-93.

6. Feuerwerker L, Sena R. Contribuição ao movimento de mudança na formaçãoprofissional em saúde: uma avaliação das experiências UNI. Interface Comunic Saúde Educ. 2002; 6(10):37-50.

7. Araújo ME, Zilbovicius C. A formação acadêmica para o trabalho no SistemaÚnico de Saúde (SUS). In: Moysés ST, Kriger L, Moysés SJ. (Orgs.). Saúde bucal das famílias: trabalhando com evidências. São Paulo: Artes Médicas, 2008. p.277-90.

8. Morita MC, Haddad AE. A concepção pedagógica e as Diretrizes CurricularesNacionais: interface da área da Educação e da Saúde na perspectiva da formação e dotrabalho das equipes da Saúde da Família. In: Moysés ST, Kriger L, Moysés SJ. (Orgs.). Saúde Bucal das Famílias: trabalhando com evidências. São Paulo: ArtesMédicas, 2008. p.268-76.

9. Ceccim RB, Ferla AA. Educação e saúde: ensino e cidadania como travessia de fronteiras. Trab Educ Saúde. 2008; 6(3):443-56.

10. Ceccim RB. Descentralização e disseminaçãode capacidade pedagógica na saúde. Ciênc Saúde Coletiva. 2005; 10(4):975-86.

11. Trajman A, Assunção N, Venturi M, Tobias D, Toschi W, Brant V. A preceptoria na rede básica da Secretaria Municipal de Saúdedo Rio de
Janeiro: opinião dos profissionais de saúde. Rev Bras Educ Méd. 2009; 33(1):24-32.

12. Haddad AE, Pierantoni CR, Ristoff D, Xavier IM, Giolo J, Silva LB (Orgs) A trajetória dos cursos de graduação na área da saúde: 19912004. Instituto Nacional de Estudos e Pesquisas Educacionais Anísio Teixeira, 2006.

13. Toassi RFC, Stobäus CD, Mosquera JJM, Moysés SJ. Currículo integrado no ensino de Odontologia: novos sentidos para a formação na área da saúde. Interface Comunic Saúde Educ. 2012; 16(41):529-42.

14. Forte FDS, Pessoa TRRF, Freitas CHSM, Pereira CAL, Carvalho JPM. Reorientação na formação de cirurgiões-dentistas: o olhar dos preceptores sobre estágios supervisionados no Sistema Único de Saúde (SUS). Interface. 2015; 1(19):831-43.

15. Neta AA, Alves MSCF. A comunidade como local de protagonismo na integração ensinoserviço e atuação multiprofissional. Trab Educ Saúde. 2016; 14(1): 221-35.

\section{Correspondência para:}

Tatiana Frederico de Almeida

E-mail: tatifrederico@yahoo.com.br

Rua Sócrates Guanaes Gomes, 44/1101 Candeal 40290-720 Salvador/BA 